\title{
An Iterative Method for Solving of Coupled Equations for Conductive-Radiative Heat Transfer in Dielectric Layers
}

\author{
Vasyl Chekurin and Yurij Boychuk \\ Pidstryhach Institute for Applied Problems of Mechanics and Mathematics, Academy of Sciences of Ukraine, 3-b Naukova Str., \\ Lviv 79060, Ukraine \\ Correspondence should be addressed to Vasyl Chekurin; v.chekurin@gmail.com
}

Received 17 March 2017; Revised 31 May 2017; Accepted 6 June 2017; Published 16 August 2017

Academic Editor: Mariano Torrisi

Copyright (C) 2017 Vasyl Chekurin and Yurij Boychuk. This is an open access article distributed under the Creative Commons Attribution License, which permits unrestricted use, distribution, and reproduction in any medium, provided the original work is properly cited.

\begin{abstract}
The mathematical model for describing combined conductive-radiative heat transfer in a dielectric layer, which emits, absorbs, and scatters IR radiation both in its volume and on the boundary, has been considered. A nonlinear stationary boundary-value problem for coupled heat and radiation transfer equations for the layer, which exchanges by energy with external medium by convection and radiation, has been formulated. In the case of optically thick layer, when its thickness is much more of photon-free path, the problem becomes a singularly perturbed one. In the inverse case of optically thin layer, the problem is regularly perturbed, and it becomes a regular (unperturbed) one, when the layer's thickness is of order of several photon-free paths. An iterative method for solving of the unperturbed problem has been developed and its convergence has been tested numerically. With the use of the method, the temperature field and radiation fluxes have been studied. The model and method can be used for development of noncontact methods for temperature testing in dielectrics and for nondestructive determination of its radiation properties on the base of the data obtained by remote measuring of IR radiation emitted by the layer.
\end{abstract}

\section{Introduction}

Necessity for studying of coupled conductive and radiative heat exchange in continuous mediums arises in various theoretical and applied disciplines: in thermal physics, in plasma physics, in solid state physics, in space technologies, in technologies of semiconductor, glass, and polymer materials, and in methodologies for thermal measurement and nondestructive testing. Various methods are used for analytical and/or numerical solving of boundary-value problems, formulated with the use of certain mathematical models describing the coupled processes of conductive and radiative heat transfer.

In the case of opaque bodies (metals), possessing high thermal conductivity, one can neglect radiative heat transfer in the body's volume and take into account only the radiative heat flux emitting by body's boundary outward. Outer IR radiation, which is incident on the boundary of such body, is totally absorbed by the boundary. In agreement with the Stefan-Boltzmann law the radiative flux is proportional to the fourth degree of surface temperature [1]. Hence we obtain in this case a nonlinear boundary-value problem for linear heat conduction equation.

Dielectric objects (nonconductors), such as glass, plastics, heat insulations, semiconductors, and biological tissues, are translucent for IR radiation. Radiation emitted by inner points of such object can spread on considerable distances in the body's volume and can stream away from the volume through the boundary. These two radiation energy fluxes can be comparable with volumetric conductive flux and surface convective flux correspondingly. Hence they should be accounted in the total energy balance.

To describe energy transfer in such mediums, the "exact" macroscopic model can be used. It includes coupled integrodifferential equations, heat conduction equation (HCE) and radiation transfer equation (RTE) [1]. They involve two unknown functions defined in the body's volume V: temperature $T(\mathbf{r}, t)$, dependent on spatial coordinates $\mathbf{r} \epsilon$ $\mathbf{V}$ and time $t$, and spectral radiation intensity $I_{\nu}(\mathbf{r}, \boldsymbol{\omega}, t)$ being a function of IR radiation frequency $v$, spatial coordinates 
$\mathbf{r} \in \mathbf{V}$, direction $\boldsymbol{\omega} \in S^{2}$, and time $t$. The model also includes coupled relations expressing the conditions, in which the temperature $T(\mathbf{r}, t)$ and intensity $I_{\nu}(\mathbf{r}, \boldsymbol{\omega}, t)$ should satisfy on the boundary $\partial \mathbf{V}$ of the body. Fundamentals of the theory for radiation transfer in participating heat conducting medium can be found in particular in the monographs [1-3].

Nonlinear interconnected integrodifferential equations and nonlinear nonlocal boundary conditions make boundary-value problems, formulated within this model, too complicated for their solving in a closed form. Furthermore, the dimensionality of the problems creates additional computational complexity: in the case that $3 \mathrm{D}$ bodies intensity $I_{\nu}(\mathbf{r}, \boldsymbol{\omega}, t)$ is dependent on seven scalar independent variables (frequency $v$, three spatial coordinates $x, y, z$, zenith $\theta$, azimuth $\varphi$ angles determining direction $\boldsymbol{\omega}$, and time $t$ ), temperature $T(\mathbf{r}, t)$ is dependent on four independent variables $(x, y, z$, and $t)$. To overcome these complications, one can make some simplifying assumptions concerning the model, restrict the consideration by $1 \mathrm{D}$ or $2 \mathrm{D}$ geometry, and apply numerical methods.

A significant simplification of the problem can be attained in the case, when the contribution of the radiative energy flux into the total medium's heat flux is a negligible quantity. It can be true at sufficiently low temperatures. In this case the problem becomes uncoupled and a linear one. In fact in this case one can treat temperature field as a prescribed function of spatial coordinate and time and concentrate his effort on solving the radiation transfer problem. Such approximation was used by many authors to study $1 \mathrm{D}$ radiation transfer problems (see, for examples, monograph [1] and [4-6]). The principal difficulties in solving the problem in this case are the presence of the scattering integral in RTE and nonlocal boundary conditions, arising when scattering of radiation by the boundary is taken into consideration.

There are several effective approaches to overcome this difficulty. Among them is the method of discrete ordinates, in agreement with which RTE is solved for a set of discrete directions, each of which is associated with a solid angle in which the intensity is assumed to be constant [7, 8]. Another method, known as spherical harmonic method, consists in a Legendre expansion of the radiation intensity and the phase function in the radiation transfer equation [4-6].

The heat transfer problems in the coupled conductiveradiative formulation are fundamentally nonlinear. Usually HCE, used in "exact" coupled problems, is formulated proceeding from the energy conservation equation [1]. Whereupon HCE contains the divergence of the total energy flux $\mathbf{q}$ including two components, conductive heat flux $\mathbf{q}_{c}$ and radiative energy flux $\mathbf{q}_{r}: \mathbf{q}=\mathbf{q}_{c}+\mathbf{q}_{r}$. Hence, the boundary conditions for such HCE should be formulated in terms of temperature $T$ and/or total energy flux q. The conductive flux can be presented, in agreement with Fourier law, as $\mathbf{q}_{r}=$ $-k_{r} \nabla T$ or with the use of generalized form, as $\tau_{0} \partial \mathbf{q}_{r} / d t+\mathbf{q}_{r}=$ $-k_{r} \nabla T$. Here $k_{c}$ is specific heat conductivity, $\nabla$ denotes the gradient operator, and $\tau_{0}$ stands for relaxation time. These two presentations lead to parabolic and hyperbolic HCE correspondingly. To make these equations well defined the flux $\mathbf{q}_{r}$ should be determined. In the "exact" formulation one can use the definition of radiation energy flux in terms of radiation intensity as [1] $q_{r}=\int_{0}^{\infty} \int_{4 \pi} \omega I_{\nu}(\mathbf{r}, \boldsymbol{\omega}, t) d \boldsymbol{\omega} d \nu$. Hence, to determine $\mathbf{q}_{r}$ one should anyway solve RTE, which, in turn, is depending on the temperature field.

With the use of an appropriate iterative process HCE and RTE can be solved consecutively.

Examples of solutions of such coupled problems for a plane layer with the use of numerical methods can be found in [6-9]. Parabolic HCE was used in [6, 9], in which temperature field in semitransparent medium subjected to a pulse irradiation was studied numerically. In [9], in particular, implicit central-difference scheme was employed for solving HCE, while a discrete curved ray-tracing method was used to solve RTE. In [7, 8], the hyperbolic HCE was used in studying of non-Fourier effects on transient coupled radiativeconductive heat transfer in one-dimensional semitransparent medium subjected to a periodic irradiation. Here hyperbolic HCE was solved by the flux-splitting method, and RTE was solved by the discrete ordinate method.

We would notice here that the results of numerical study presented, in particular, in [9], were obtained for the layer of optical thickness $\bar{h}=0.1$, that is, for optically thin medium. In [8], where the influence of $\bar{h}$ on temperature field in the layer was studied, the maximum used value of optical thickness was taken to be equal to 1.0 .

As we will show further, the problem in coupled "exact" formulation can be solved with the use of conventional method only for layers with optical thickness $\bar{h}$ of order unity or smaller. The reason is the presence of two spatial scales in the problem: the layer's physical thickness $h$ and the photon mean free path $l_{0}$. As a consequence, the coefficient at the highest spatial derivative in HCE decreases with increasing of $\bar{h} \equiv h / l_{0}$ as $1 / \bar{h}^{2}$. This becomes apparent in occurrence of so-called boundary layer of thickness of order $l_{0}$ at sufficiently large $\bar{h}$. The temperature and radiation intensity are sharply varied in the boundary layer. Whereupon the problem becomes singularly disturbed at large $\bar{h}$, special approaches should be used to analyze it numerically [10].

But some problems for optically thick layers can be solved numerically with the use of an approach, which known as Rosseland's or diffusion approximation $[1,3]$. In this approximation, radiation flux $\mathbf{q}_{r}$ is also taken to be proportional to temperature gradient $\mathbf{q}_{r}=-k_{r} \nabla T$, where $k_{r}$ is radiation conductivity, which is defined as $k_{r}=16 / 3 \sigma_{\mathrm{SB}} T^{3} / \kappa$ [3], and $\sigma_{\mathrm{SB}}$ stands for the Stefan-Boltzmann constant $\sigma_{\mathrm{SB}} \approx$ $5.670367 \times 10^{-8} \mathrm{~W} \cdot \mathrm{m}^{2} \cdot \mathrm{K}^{-4}$.

Hence, applying the diffusion approximation, we exclude RTE from the problems of conductive-radiative heat exchange. Now we can deal with HCE for the medium with the modified heat conductivity defined as $k=k_{c}+k_{r}$, which depends on the temperature. As a consequence, the second spatial scale $l_{0}$ also vanishes, and the problem remains regular at arbitrary optical thickness $\bar{h}$.

This approach was successfully used in various practical problems, in particular, for evaluation of effective heat resistance properties of low-conductivity coats [11-13]. But it is important to realize that the diffusive approximation is valid only at sufficiently low temperature gradients and only in 
inner medium's domains, remote from the boundary $\partial \mathbf{V}$ on distance exceeding the mean free path $l_{0}$. In a boundary layer with the thickness of order $l_{0}$ this approach does not work.

According to this approximation the IR radiation streaming out the object into external medium is determined solely by its surface temperature, whereas the radiation of the boundary layer's points is not take into account.

But there are problems in which the exact value of IR energy, emitted by the object, is significant. In this connection, we can mention, as an example, IR thermography and its application to determination of temperature fields of solid objects [14, 15]. In the case of opaque bodies (metals), the IR radiation streaming away from the body is totally emitted by its surface. So, measuring radiosity of the target object, one can unambiguously judge about its surface temperature. But in the case of dielectric objects it is not so. Outgoing radiation energy flux in this case contains two constituents: the radiation emitted by the surface and the flux, emitted in the boundary layer, which passes through the surface outward. Now, to interpret the measurement data, one should separate the constituents in the total measuring flux. Obviously the needed calculation cannot be performed to be sufficiently exact with the use of diffusion model.

This also applies to the model considered in [7-9], in which HCE is obtained starting from the total energy conservation law and the assumption about black boundary was applied. This assumption enabled satisfying simultaneously boundary conditions both for total energy flux $\mathbf{q}$ and for radiation intensity and to simplify the computational procedure. But many of real world objects possess the boundary, which is not absolutely black. It not only absorbs and emits IR radiation but also reflects and transmits it. Hence, this model, as well as the diffusion approximation, cannot be applied to such objects and it cannot be used for the inverse problems mentioned before.

The objectives of the paper are mathematical formulation of coupled problem for combined conductive-radiative heat transfer in dielectric layer of participating medium, which emits, absorbs, and scatters IR radiation in the volume and on the boundary; qualitative study of formulated problem depending on thermophysical and radiative properties of medium and layer's thickness; developing an iterative method for solving the coupled problem and studying of its convergence.

\section{Governing Equations}

A plane isotropic and physically homogeneous dielectric layer $\mathbf{B}$ occupying a region $\mathbf{V}=\{(x, y, z): x \in$ $(-\infty, \infty), \quad y \in(-b, b), z \in(-\infty, \infty)\}$ is considered. Here $b>0$ is the layer's half-thickness. The body $\mathbf{B}$ is considered as a thermodynamic system consisting of two interacting subsystems, the participating medium and IR radiation, which occupy the same region $\mathbf{V}$. The interaction of the subsystems is realized by absorbing, emitting, and coherent scattering of IR radiation by the layer's medium both in the volume $\mathbf{V}$ and on its boundary $\partial \mathbf{V}=\mathbf{S}_{1} \cup \mathbf{S}_{2}$. Here $\mathbf{S}_{1}=\{(x, y, z): x \in(-\infty, \infty), y=b, \quad z \in(-\infty, \infty)\}, \mathbf{S}_{2}=$ $\{(x, y, z): x \in(-\infty, \infty), y=-b, \quad z \in(-\infty, \infty)\}$.
Interaction of the subsystems in the volume $\mathbf{V}$ is defined by volumetric radiative parameters: the spectral refraction index $n_{v}$, spectral absorption $\kappa_{v}^{a}$, and emitting $\kappa_{v}^{e}$ and scattering $\sigma_{\nu}$ coefficients (further we suppose $\kappa_{v}^{e}=\kappa_{\nu}^{a} \equiv \kappa_{\nu}$ ) and by the spectral phase function $p_{\nu}\left(\mu, \mu^{\prime}\right)$. Interaction of the subsystems on the boundary $\partial \mathbf{V}$ is defined by its surface radiative parameters: the spectral absorptivity/emittivity $\varepsilon_{v}$ and reflectivity $\rho_{v}$.

The layer is situated within nonparticipating external medium with prescribed temperature $T^{\text {ext }}$ and optical refraction index $n=1$. The subsystems exchange by energy with external medium by convection, emitting, and absorbing of the radiation through the boundary $\partial \mathbf{V}$. The energy exchange of the layer's medium with the external medium through the boundary $\partial \mathbf{V}$ is defined by material parameters, coefficient of convective heat exchange, and by spectral absorptivity/emittivity $\varepsilon_{v}$. The energy exchange of the layer's radiation with the external medium through the boundary $\partial \mathbf{V}$ is defined by boundary's spectral transmissivity $\tau_{v}$.

1D stationary thermal state of the layer's medium is identically determined by temperature $T(y)$, depending on transversal coordinate $y \in[-b, b]$. The state of the layer's radiation is identically determined by spectral intensity or radiation $I_{\lambda}(y, \mu)$, depending on $y$ and direction of radiation's propagation. Here $\mu \in[-1,1], \mu \equiv \cos \theta$, where $\theta$ is the angle between a direction and axes $y$.

Under such consideration we can use the equation of energy balance in the layer's medium to obtain HCE:

$$
\begin{aligned}
- & \frac{d}{d y}\left(k_{c} \frac{d T(y)}{d y}\right) \\
& =2 \pi \int_{-1}^{1} \int_{0}^{\infty} \kappa_{\nu}\left(I_{\nu}(y, \mu)-n_{\nu}^{2} I_{\nu b}(T(y))\right) d \nu d \mu .
\end{aligned}
$$

Here $k_{c}$ stands for thermal conductivity of the layer's medium.

This equation, unlike HCE, used in [7-9] and others, do not contain the radiation heat flux $q_{r}$. But instead it contains the source term in its right-hand side which takes into consideration the interaction of the layer's medium with the medium's radiation.

Radiation intensity $I_{\nu}(y, \mu)$ satisfies, in the region $\mathbf{V}$,

$$
\begin{aligned}
\mu & \frac{\partial I_{\nu}(y, \mu)}{\partial y}+\beta_{\nu} I_{\nu}(y, \mu) \\
= & \kappa_{\nu} n_{\nu}^{2} I_{\nu b}(T(y)) \\
& \quad+\frac{1}{2} \sigma_{\nu} \int_{-1}^{1} p_{\nu}\left(\mu^{\prime}, \mu\right) I_{\nu}\left(y, \mu^{\prime}\right) d \mu^{\prime},
\end{aligned}
$$

where $\beta_{v}=\kappa_{v}+\sigma_{v}$ stands for spectral extinction coefficient.

It can be easily shown, that, proceeding from (1) and (2), HCE, used in [7-9], can be obtained. To do this, one should integrate (2) with respect to variables $\mu \in[-1,1]$ and $\varphi \in$ $[0,2 \pi]$ and sum the obtained equation with (1). 
The spectral phase function $p_{\nu}\left(\mu, \mu^{\prime}\right)$ in (2) determines the fraction of radiation incident in direction $\mu^{\prime}$ and scattered into direction $\mu$. It satisfies the normality condition [1]:

$$
\frac{1}{2} \int_{-1}^{1} p\left(\mu^{\prime}, \mu\right) d \mu=1
$$

Planck function,

$$
I_{\nu b}(T) \equiv \frac{2 h v^{3}}{c_{0}\left[\exp \left(h v / k_{B} T\right)-1\right]},
$$

in (1) and (2) determines the intensity of blackbody's radiation in vacuum. Here $h \simeq 6.626075 \mathrm{~J} \cdot \mathrm{s}$ is Planck constant, $k_{B} \simeq 1.380658 \mathrm{~J} / \mathrm{K}$ is Boltzmann constant, and $c_{0} \simeq$ $2.99792458 \mathrm{~m} / \mathrm{s}$ is speed of light in vacuum.

Constituents $Q^{e}(T)$ and $Q^{a}\left(I_{\nu}\right)$

$$
\begin{aligned}
& Q^{e}(T) \equiv 2 \pi \int_{-1}^{1} \int_{0}^{\infty} \kappa_{\nu} n_{\nu}{ }^{2} I_{\nu b}(T(y)) d \nu d \mu, \\
& Q^{a}\left(I_{\nu}\right) \equiv 2 \pi \int_{-1}^{1} \int_{0}^{\infty} \kappa_{\nu} I_{\nu}(y, \mu) d \nu d \mu,
\end{aligned}
$$

in right-hand side of (1) determine, respectively, the local rates of radiation energy emitted and absorbing by the medium in volume $\mathbf{V}$.

Terms $S_{v}^{\text {sc }}$ and $S_{\nu}^{e}(T)$

$$
\begin{aligned}
S_{\nu}^{\mathrm{sc}}\left(I_{\nu}\right) & =\frac{1}{2} \sigma_{\nu} \int_{-1}^{1} p\left(\mu^{\prime}, \mu\right) I_{\nu}\left(y, \mu^{\prime}\right) d \mu^{\prime}, \\
S_{\nu}^{e}(T) & =n_{\nu}{ }^{2} \kappa_{\nu} I_{v b}(T),
\end{aligned}
$$

in right-hand side of (2) are the scattering integral and radiation inflow caused by its emitting by the medium in the volume $\mathbf{V}$.

We consider the boundary $\partial \mathbf{V}$ as translucent one, which diffusely emits, scatters, and absorbs IR radiation.

Radiation with intensity $\left.I_{\nu}(\mu)\right|_{y=b}, \mu \in(0,1)$ and $\left.I_{\nu}^{\text {ext }}(\mu)\right|_{y=b}, \quad \mu \in(-1,0)$, incident on the boundary plane $\mathbf{S}_{1}$ out of the volume $\mathbf{V}$ and from the external medium, is partly scattered back, partly absorbed by the boundary $\mathbf{S}_{1}$, and partly transmitted through this boundary. Let $\rho_{1 v} \in$ $[0,1]$ be a spectral hemispherical reflectivity of the boundary $\mathbf{S}_{1} ; \varepsilon_{1 v} \in[0,1]$ is its spectral hemispherical absorptivity. Then $\rho_{1 v}$ and $\varepsilon_{1 \nu}\left(1-\rho_{1 \nu}\right)$ are the fraction of radiation scattered back and absorbed by the boundary $\mathbf{S}_{1}$, whereas the part of transmitted radiation will be equal to $\tau_{1 v} \equiv 1-\rho_{1 v}-\varepsilon_{1 \nu}(1-$ $\left.\rho_{1 \nu}\right)=\left(1-\varepsilon_{1 \nu}\right)\left(1-\rho_{1 \nu}\right)$.

Similarly the boundary plane $\mathbf{S}_{2}$, which is characterized by the radiative properties $\rho_{2 v}, \varepsilon_{2 v}$, and $\tau_{2 \nu}=\left(1-\varepsilon_{2 v}\right)\left(1-\rho_{2 v}\right)$, scatters, absorbs, and transmits the radiation of intensities $\left.I_{\nu}(\mu)\right|_{y=-b}, \quad \mu \in(-1,0)$ and $\left.I_{\nu}^{\text {ext }}(\mu)\right|_{y=-b}, \quad \mu \in(0,1)$ incident on it from the volume $\mathbf{V}$ and external medium correspondingly.

The intensities of radiation emitting by the planes $\mathbf{S}_{1}, \mathbf{S}_{2}$ into the volume $\mathbf{V}$ and in external medium are defined as $I_{1 v}^{e, \text { out }}(\mu)=\varepsilon_{1} I_{\nu B}\left(\left.T\right|_{y=b}\right), \quad \mu \in(0,1)$ and $I_{1 v}^{e, \text { in }}(\mu)=$ $\varepsilon_{1} n_{v}^{2} I_{\nu B}\left(\left.T\right|_{y=b}\right), \mu \in(-1,0), I_{2 v}^{e, \text { out }}(\mu)=\varepsilon_{2} I_{v B}\left(\left.T\right|_{y=-b}\right), \mu \in$
$(-1,0)$ and $I_{2 \nu}^{e, \text { in }}(\mu)=\varepsilon_{2} n^{2} I_{\nu B}\left(\left.T\right|_{y=-b}\right), \mu \in(0,1)$, correspondingly.

Now, applying the energy conservation law, we can establish the conditions (7) and (8), which dependent variables $T(y)$ and $I_{\nu}(y, \mu)$ satisfy on the boundary planes $\mathbf{S}_{1}$ and $\mathbf{S}_{2}$ :

$$
\begin{aligned}
& -\left.k_{c} \frac{d T}{d y}\right|_{y=b_{k}} \\
& =-h_{1}\left(\left.T\right|_{y=b_{k}}-\left.T_{\text {ext }}\right|_{y=b_{k}}\right) \\
& -\pi \int_{0}^{\infty}\left(1+n_{\nu}^{2}\right) \varepsilon_{k v} I_{v b}\left(\left.T\right|_{y=b_{k}}\right) d \nu \\
& +\left.2 \pi \int_{\mu \in m_{k}} \int_{0}^{\infty} \varepsilon_{k \nu}\left(1-\rho_{k \nu}\right) I_{\nu}(\mu)\right|_{y=b_{k}} d \nu \mu d \mu \\
& +\left.2 \pi \int_{\mu \in m_{l}} \int_{0}^{\infty} \varepsilon_{k \nu}\left(1-\rho_{k \nu}\right) I_{\nu}^{\mathrm{ext}}(\mu)\right|_{y=b_{k}} d \nu \mu d \mu \\
& \left.I_{\nu}(\mu)\right|_{y=b_{k}} \\
& =n_{\nu}^{2} \varepsilon_{k v} I_{v b}\left(\left.T\right|_{y=b_{k}}\right)+\left.\rho_{k v} \int_{\mu^{\prime} \in m_{k}} I_{\nu}\left(\mu^{\prime}\right)\right|_{y=b_{k}} d \mu^{\prime} \\
& +\left.\left(1-\varepsilon_{k \nu}\right)\left(1-\rho_{k \nu}\right) \int_{\mu^{\prime} \in m_{l}} I_{\nu}^{\mathrm{ext}}\left(\mu^{\prime}\right)\right|_{y=b_{k}} d \mu^{\prime},
\end{aligned}
$$

$\mu \in m_{l}$.

Here we used denotations: $k, l=1,2 ; l \neq k, b_{1}=b, b_{2}=-b$. $m_{1}=(0,1), m_{2}=(-1,0)$.

Unlike [7-9], boundary conditions (7) for HCE in our case do not contain the radiation heat flux $\mathbf{q}_{r}$. The first and second terms in the right-hand parts of this relation are the surface energy loss caused correspondingly by the convection and the radiative emission of the boundary plane $S_{k}$. The third and fourth members take into account the energy of internal and external radiation incident on the boundary plane $S_{k}$ and absorbed by it.

The first terms in the right-hand side of the relations (8) determine spectral intensities of the radiation emitting by the boundaries $\mathbf{S}_{k}$ into the volume $\mathbf{V}$, the second ones are the spectral intensities of IR radiation, reflected by boundary $\mathbf{S}_{k}$ into the volume $\mathbf{V}$, and the last one is intensities of the external radiation penetrating through the boundaries $\mathbf{S}_{k}$ into the volume $\mathbf{V}$.

The spectral intensities of radiation proceeding out from layer $\mathbf{B}$ through the planes $\mathbf{S}_{k}$ (layer's spectral radiosity) can be determined as

$$
\begin{aligned}
I_{k \nu}^{\text {out }}(\mu)= & \varepsilon_{k v} I_{v b}\left(\left.T\right|_{y=b_{k}}\right) \\
& +\left.\left(1-\varepsilon_{k v}\right)\left(1-\rho_{k v}\right) I_{\nu}(\mu)\right|_{y=b_{k}} \\
& +\left.\rho_{k v} \int_{\mu^{\prime} \in m_{l}} I_{\nu}^{\mathrm{ext}}\left(\mu^{\prime}\right)\right|_{y=b_{k}} d \mu^{\prime}, \quad \mu \in m_{k} .
\end{aligned}
$$

The second term in the right-hand part of the relations (9) takes into account the internal radiation, passing through the boundary into the external medium, whereas the last 
term in this part determines the spectral intensities of the external radiation, reflecting back into external medium by the boundary.

Energy flux, flowing-off layer $\mathbf{B}$ through boundary $\mathbf{S}_{k}$, can be determined as

$$
\begin{aligned}
q_{k}^{\text {out }}= & h\left(\left.T\right|_{y=b_{k}}-\left.T^{\text {ext }}\right|_{y=b_{k}}\right) \\
& +2 \pi \int_{\mu \in m_{k}} \int_{0}^{\infty} I_{1 \nu}^{\text {out }} d \nu \mu d \mu .
\end{aligned}
$$

At the given values $\left.T^{\text {ext }}\right|_{y=b_{k}}$ and $\left.I_{v}^{\text {ext }}(\mu)\right|_{y=b_{k}}$ of external temperature and radiation intensity on the boundary planes, given thermal parameters $k_{c}, h$, radiative properties of layer's medium $\kappa_{v}, \sigma_{v}, p\left(\mu^{\prime}, \mu\right)$, and $\varepsilon_{k v}, \rho_{k v}$ of its boundary $\partial \mathbf{V}$, (1) and (2) together with boundary conditions (7) and (8) determine correct formulation of direct boundary-value problem for combined conductive-radiative heat exchange in layer $\mathbf{B}$, which exchanges by energy with external medium by convection and radiation.

Solution of this problem enables determining, with the use of relations (9) and (10), the angle distribution of radiation intensity and total energy flux emitting by the layer into the external medium. These parameters are important when inverse problems for noncontact determination of temperature field in the layer and/or radiative parameters of the layer's medium are considered.

In further consideration, we will apply the approximation of "gray" medium to the layer $[1,3]$. This does not change the types of (1) and (2) and boundary conditions (7), (8) but permits substantially reducing the amount of data needed for definition of the radiative properties of layer $\mathbf{B}$. Additionally we will restrict the consideration by the case of isotropic scattering, assuming the phase function $p\left(\mu^{\prime}, \mu\right)=1[1]$ and consider thermal and radiative properties as independent of temperature $T$. In this approximation (1) and (2) and boundary conditions (7) and (8) take the forms (11) and (12) and (16)-(19), respectively:

$$
\begin{aligned}
& k_{c} \frac{d^{2} T(y)}{d y^{2}}=2 \pi \kappa \int_{-1}^{1}\left(n^{2} I_{b}(T(y))-I(y, \mu)\right) d \mu \\
& \mu \frac{1}{\beta} \frac{\partial I(y, \mu)}{\partial y}+I(y, \mu) \\
& =\bar{\kappa} n^{2} I_{b}(T(y))+\frac{1}{2} \bar{\sigma} \int_{-1}^{1} I\left(y, \mu^{\prime}\right) d \mu^{\prime} \\
& -\left.k_{c} \frac{d T}{d y}\right|_{y=b_{k}} \\
& =-h_{k}\left(\left.T\right|_{y=b_{k}}-\left.T^{\operatorname{ext}}\right|_{y=b_{k}}\right) \\
& \quad-\pi\left(1+n^{-2}\right) \varepsilon_{k} I_{b}\left(\left.T\right|_{y=b_{k}}\right) \\
& +\left.2 \pi \varepsilon_{k}\left(1-\rho_{k}\right) \int_{\mu \in m_{k}} I(\mu)\right|_{y=b_{k}} \mu d \mu
\end{aligned}
$$

$$
+\left.2 \pi \varepsilon_{k}\left(1-\rho_{k}\right) \int_{\mu \in m_{l}} I^{\mathrm{ext}}(\mu)\right|_{y=b} \mu d \mu,
$$

$$
\begin{aligned}
& \left.I(\mu)\right|_{y=b_{k}} \\
& =\varepsilon_{k} I_{b}\left(\left.T\right|_{y=b_{k}}\right)+\left.\rho_{k} \int_{\mu^{\prime} \in m_{k}} I\left(\mu^{\prime}\right)\right|_{y=b_{k}} d \mu^{\prime} \\
& \quad+\left.\left(1-\varepsilon_{k}\right)\left(1-\rho_{k}\right) \int_{\mu^{\prime} \in m_{l}} I^{\mathrm{ext}}\left(\mu^{\prime}\right)\right|_{y=b_{k}} d \mu^{\prime},
\end{aligned}
$$

$$
\mu \in m_{l} \text {. }
$$

Here $I_{b}(T) \equiv n^{2} \sigma_{\mathrm{SB}} T^{4} / \pi, n$ is integral refraction index, $\kappa$ and $\sigma$ stand for integral absorption and scattering coefficients, $\beta \equiv \kappa+\sigma$ is integral extinction coefficient, $\bar{\sigma} \equiv \sigma / \beta$ stands for scattering albedo, $\bar{\kappa} \equiv \kappa / \beta=(1-\bar{\sigma})$, and $\rho_{k}$ and $\varepsilon_{k}$ are hemispherical total reflectivity and hemispherical total absorptivity of the boundary $\mathbf{S}_{k} ; I(y, \mu)$ and $I^{\text {ext }}(\mu)$ are the integral intensities of internal and external radiation.

Dimensionless parameters $\bar{\kappa} \in[0,1], \bar{\sigma} \in[0,1], \bar{\kappa}+\bar{\sigma}=1$. We will consider participating media for which $\bar{\kappa}=O(1), \bar{\sigma}=$ $O(1)$.

The integral radiosity of the layer $\mathbf{B}$ on the outer side of its boundary plane $\mathbf{S}_{k}$ takes the forms in this case:

$$
\begin{aligned}
I_{k}^{\text {out }}(\mu)= & \varepsilon_{k} n^{-2} I_{b}\left(\left.T\right|_{y=b_{k}}\right) \\
& +\left.\left(1-\varepsilon_{k}\right)\left(1-\rho_{k}\right) I\left(\mu^{\prime}\right)\right|_{y=b_{k}} \\
& +\left.\rho_{k} \int_{\mu^{\prime} \in m_{l}} I^{\text {ext }}\left(\mu^{\prime}\right)\right|_{y=b_{k}} d \mu^{\prime}, \quad \mu \in m_{k} .
\end{aligned}
$$

\section{Analysis of the Problem}

At small $\mu$ (12) is singularly perturbed as at $\mu=0$ its type is being changed. So we will consider function $I(y, \mu)$ as piecewise homogeneous with respect to variable $\mu$ :

$$
I(y, \mu)= \begin{cases}I_{1}(y, \mu), & \mu \in(0,1) \\ I_{2}(y, \mu), & \mu \in(-1,0) .\end{cases}
$$

Due to this we will consider instead of (12) the coupled system

$$
\begin{aligned}
& \mu \frac{1}{\beta} \frac{\partial I_{1}(y, \mu)}{\partial y}+I_{1}(y, \mu) \\
&= \bar{\kappa} I_{b}(T(y)) \\
& \quad+\frac{1}{2} \bar{\sigma}\left(\int_{0}^{1} I_{1}\left(y, \mu^{\prime}\right) d \mu^{\prime}+\int_{-1}^{0} I_{2}\left(y, \mu^{\prime}\right) d \mu^{\prime}\right), \\
& \mu \frac{1}{\beta} \frac{\partial I_{2}(y, \mu)}{\partial y}+I_{2}(y, \mu) \\
&=\bar{\kappa} I_{b}(T(y)) \\
& \quad+\frac{1}{2} \bar{\sigma}\left(\int_{0}^{1} I_{1}\left(y, \mu^{\prime}\right) d \mu^{\prime}+\int_{-1}^{0} I_{2}\left(y, \mu^{\prime}\right) d \mu^{\prime}\right) .
\end{aligned}
$$


In the new denotations boundary conditions (14) for (17), (18) take the following form:

$$
\begin{aligned}
\left.I_{k}(\mu)\right|_{y=b_{l}}= & \varepsilon_{l} I_{b}\left(\left.T\right|_{y=b_{l}}\right)+\left.\rho_{l} \int_{\mu^{\prime} \in m_{l}} I_{l}\left(\mu^{\prime}\right)\right|_{y=b_{l}} d \mu^{\prime} \\
& +\left(1-\varepsilon_{l}\right)\left(1-\rho_{l}\right) \int_{\mu \in m_{k}} I_{l}^{\mathrm{ext}}\left(\mu^{\prime}\right) d \mu^{\prime} .
\end{aligned}
$$

Here $\left.I_{k}^{\text {ext }}(\mu) \equiv I^{\text {ext }}(\mu)\right|_{y=b_{k}}$

We also rewrite (11) in equivalent form:

$$
\begin{aligned}
& k_{c} \frac{d^{2} T(y)}{d y^{2}}=2 \pi \kappa\left(2 I_{b}(T(y))\right. \\
& \left.\quad-\left(\int_{0}^{1} I_{1}(y, \mu) d \mu+\int_{-1}^{0} I_{2}(y, \mu) d \mu\right)\right) .
\end{aligned}
$$

The nonlinear second-order differential (20) should be subordinated to both boundary conditions (13). With the use of the new dependent variables these conditions take the following forms:

$$
\begin{aligned}
& -\left.k_{c} \frac{d T}{d y}\right|_{y=b_{k}}-h_{k}\left(\left.T\right|_{y=b_{k}}-T_{k}^{\mathrm{ext}}\right) \\
& -\pi\left(1+n^{-2}\right) \varepsilon_{k} I_{b}\left(\left.T\right|_{y=b_{k}}\right) \\
& +\left.2 \pi \varepsilon_{k}\left(1-\rho_{k}\right) \int_{\mu \in m_{k}} I_{k}(\mu)\right|_{y=b_{k}} \mu d \mu \\
& +\left.2 \pi \varepsilon_{k}\left(1-\rho_{k}\right) \int_{\mu \in m_{l}} I_{k}^{\mathrm{ext}}(\mu)\right|_{y=b_{k}} \mu d \mu .
\end{aligned}
$$

We introduce dimensionless variables: coordinate $\bar{y}=$ $y / b$, temperature $\bar{T}(\bar{y})=T(b \bar{y}) / T_{0}$, and intensity $J(\bar{y}, \mu)=$ $I(b \bar{y}, \mu) / I_{b}\left(T_{0}\right)$, where $T_{0}$ is a characteristic temperature. With these variables equations (20), (17), and (18) and boundary conditions (21), (19) can be rewritten as

$$
\begin{aligned}
& \varepsilon_{0}^{2} \frac{d^{2} \bar{T}(\bar{y})}{d \bar{y}^{2}}=2 \frac{\bar{\kappa}^{2}}{\bar{k}}\left(2 \bar{T}(\bar{y})^{4}\right. \\
& \left.\quad-\left(\int_{0}^{1} J_{1}(\bar{y}, \mu) d \mu+\int_{-1}^{0} J_{2}(\bar{y}, \mu) d \mu\right)\right) \\
& \mu \varepsilon_{0} \frac{\partial J_{k}(\bar{y}, \mu)}{\partial \bar{y}}+J_{k}(\bar{y}, \mu)=\bar{\kappa}^{4}(\bar{y})+\frac{1}{2} \\
& \cdot \bar{\sigma}\left(\int_{0}^{1} J_{1}\left(\bar{y}, \mu^{\prime}\right) d \mu^{\prime}+\int_{-1}^{0} J_{2}\left(\bar{y}, \mu^{\prime}\right) d \mu^{\prime}\right)
\end{aligned}
$$

$$
\begin{aligned}
& -\left.\bar{k} \varepsilon_{0} \frac{d \bar{T}}{d \bar{y}}\right|_{\bar{y}= \pm 1}=-\bar{h}_{k} \bar{\kappa}\left(\left.\bar{T}\right|_{\bar{y}= \pm 1}-\left.\bar{T}^{\mathrm{ext}}\right|_{\bar{y}= \pm 1}\right)-(1 \\
& \left.+n^{-2}\right)\left.\bar{\kappa} \varepsilon_{k} \bar{T}^{4}\right|_{\bar{y}= \pm 1}+2 \varepsilon_{k}\left(1-\rho_{k}\right) \\
& \left.\cdot \bar{\kappa} \int_{\mu \in m_{k}} J_{k}(\mu)\right|_{\bar{y}= \pm 1} \mu d \mu+2 \varepsilon_{k}\left(1-\rho_{k}\right) \\
& \left.\cdot \bar{\kappa} \int_{\mu \in m_{l}} J_{k}^{\mathrm{ext}}(\mu)\right|_{\bar{y}= \pm 1} \mu d \mu, \\
& \left.J_{k}(\mu)\right|_{\bar{y}=\mp 1}=\left.\varepsilon_{l} \bar{T}^{4}\right|_{\bar{y}=\mp 1}+\left.\rho_{l} \int_{\mu^{\prime} \in m_{l}} J_{l}\left(\mu^{\prime}\right)\right|_{\bar{y}=\mp 1} d \mu^{\prime} \\
& +\left(1-\varepsilon_{l}\right)\left(1-\rho_{l}\right) \int_{\mu^{\prime} \in m_{k}} J_{l}^{\mathrm{ext}}\left(\mu^{\prime}\right) d \mu^{\prime} .
\end{aligned}
$$

Here $\varepsilon_{0} \equiv 1 / \beta b=l_{0} / b, \bar{k} \equiv k_{c} \kappa / n^{2} \sigma_{\mathrm{SB}} T_{0}^{3}, \bar{h}_{1} \equiv h_{1} / n^{2} \sigma_{\mathrm{SB}} T_{0}{ }^{3}$. In relations (24) and (25), containing the expression $y= \pm 1$ or $y=\mp 1$, the upper sign should be taken at $k=1$, whereas the lower one - should be taken at $k=2$.

Since $n^{2}=O(1), \sigma_{\mathrm{SB}} T_{0}^{3} / \kappa \sim k_{r}\left(T_{0}\right)$, where $k_{r}(T)$ is the radiative conductivity [3], $\bar{k}=O\left(k_{c} / k_{r}\left(T_{0}\right)\right)$, so dimensionless parameter $\bar{k}$ determines the contributions of conductive and radiative heat transfers in total heat flux: when $\bar{k} \gg 1$, the radiation practically does not affect temperature field in the layer. But in the case of $\bar{k}=O(1)$, the contributions of both mechanisms of heat transfer in the layer are comparable. Further we suppose the case $\bar{k}=O(1)$.

Parameter $\varepsilon_{0}$ determines the ratio of photon's mean free path $l_{0} \equiv 1 / \beta$ to layer's half-thickness $b$. Hence for layers with $b \gg l_{0}, \varepsilon_{0}$ is a small parameter. Value of extinction coefficients $\beta$ for solid dielectrics varies in the bounds $(500 \cdots 10000) \mathrm{m}^{-1}$. Hence the relation $b \gg l_{0}$ becomes valid for layers with $b \sim$ $(1 \cdots 10) \mathrm{mm}$ already. So, we can conclude, that, at $b \gg l_{0}$ (the case of optically thick layer), (22)-(23) contain the small parameter at highest derivative; hence the problem (22)-(25) is singularly perturbed [10].

In the case of optically thin layer, when $\varepsilon_{0} \gg 1$, the problem becomes regularly perturbed, and it can be solved by expansion in small parameter $1 / \varepsilon_{0}[10]$.

The case $\varepsilon_{0} \ll 1$ can be effectively analyzed quantitatively with the use of Rosseland's approximation [1]. But, as it was mentioned before, this approximation does not describe a temperature distribution and radiation fluxes within the boundary layers of thickness about $l_{0}$.

There are various methods for quantitative analysis of singularly perturbed problems [10]. One of the approaches to regularize the problems is considering them in the interior of domain and in the boundary layer separately with consequent joining of the two obtained solutions.

To implement such approach for problem (30)-(36) it is necessary to develop a method for its numerical solving for layers with thickness about photon mean path $l_{0}$. In this case $\varepsilon_{0}=O(1)$ and the problem becomes regular (unperturbed). It is convenient to use now the length $l_{0}$ as the spatial scale. 
Introducing dimensionless spatial coordinate $\xi=\beta y=y / l_{0}$, we rewrite the problem (30)-(36) in the following form:

$$
\begin{aligned}
& \frac{d^{2} \bar{T}(\xi)}{d \xi^{2}}=2 \frac{\bar{\kappa}}{\bar{\lambda}}\left(2(\bar{T}(\xi))^{4}\right. \\
& \left.-\left(\int_{0}^{1} J_{1}(\xi, \mu) d \mu+\int_{-1}^{0} J_{2}(\xi, \mu) d \mu\right)\right) \\
& \mu \frac{\partial J_{k}(\xi, \mu)}{\partial \xi}+J_{k}(\xi, \mu)=\bar{\kappa}(\bar{T}(\xi))^{4}+\frac{1}{2} \\
& \cdot \bar{\sigma}\left(\int_{0}^{1} J_{1}\left(\xi, \mu^{\prime}\right) d \mu^{\prime}+\int_{-1}^{0} J_{2}\left(\xi, \mu^{\prime}\right) d \mu^{\prime}\right) \\
& -\left.\frac{d \bar{T}}{d \xi}\right|_{\xi=\bar{b}_{k}}=-\bar{\gamma}_{k}\left(\left.\bar{T}\right|_{\xi=\bar{b}_{k}}-\left.\bar{T}^{\mathrm{ext}}\right|_{\xi=\bar{b}_{k}}\right)-\left(1+n^{-2}\right) \\
& \left.\cdot \bar{\lambda}^{-1} \varepsilon_{k} \bar{T}^{4}\right|_{\xi=\bar{b}_{k}}+2 \varepsilon_{k}\left(1-\rho_{k}\right) \\
& \left.\cdot \bar{\lambda}^{-1} \int_{\mu \in m_{k}} J_{k}(\mu)\right|_{\xi=\bar{b}_{k}} \mu d \mu+2 \varepsilon_{k}\left(1-\rho_{k}\right) \\
& \left.\cdot \bar{\lambda}^{-1} \int_{\mu \in m_{l}} J_{k}^{\mathrm{ext}}(\mu)\right|_{\xi=\bar{b}_{k}} \mu d \mu, \\
& \left.J_{k}(\mu)\right|_{\xi=\bar{b}_{l}}=\left.\varepsilon_{l} \bar{T}^{4}\right|_{\xi=\bar{b}_{l}}+\left.\rho_{l} \int_{\mu^{\prime} \in m_{l}} J_{l}\left(\mu^{\prime}\right)\right|_{\xi=\bar{b}_{l}} d \mu^{\prime}+(1 \\
& \left.-\varepsilon_{l}\right)\left(1-\rho_{l}\right) \int_{\mu^{\prime} \in m_{k}} J_{l}^{\mathrm{ext}}\left(\mu^{\prime}\right) d \mu^{\prime},
\end{aligned}
$$

where $\bar{\lambda} \equiv k_{c} \beta /\left(n^{2} \sigma_{\mathrm{SB}} T_{0}^{3}\right), \bar{b}_{1}=\bar{b}, \bar{b}_{2}=-\bar{b}, \bar{b} \equiv \beta b=$ $b / l_{0}, \gamma_{1} \equiv h_{1} / k_{c} \beta, \gamma_{2} \equiv h_{2} / k_{c} \beta$.

\section{An Iterative Method for Solving of the Unperturbed Problem}

We will develop an iterative method for solving the problem (26)-(29). We assume $J^{\text {ext }} \equiv 0$ and modify the boundary conditions (28), (29) for $k=2$. We suppose boundary $\boldsymbol{S}_{2}$ as a blackbody with prescribed fixed temperature $T_{0}$, what enables us to rewrite them in the following form:

$$
\begin{array}{r}
\left.\bar{T}\right|_{\xi=-\bar{b}}=1, \\
\left.J_{1}(\mu)\right|_{\xi=-\bar{b}}=1 .
\end{array}
$$

The boundary conditions (28) and (29) for $k=1$ remain in the following form:

$$
\begin{aligned}
-\left.\frac{d \bar{T}}{d \xi}\right|_{\xi=\bar{b}}= & -\bar{\gamma}_{1}\left(\left.\bar{T}\right|_{\xi=\bar{b}}-\left.\bar{T}^{\mathrm{ext}}\right|_{\xi=\bar{b}}\right) \\
& -\left.\left(1+n^{-2}\right) \bar{\lambda}^{-1} \varepsilon_{1} \bar{T}^{4}\right|_{\xi=\bar{b}}
\end{aligned}
$$

$$
+\left.2 \varepsilon_{1}\left(1-\rho_{1}\right) \bar{\lambda}^{-1} \int_{0}^{1} J_{1}(\mu)\right|_{\xi=\bar{b}} \mu d \mu .
$$

$$
\left.J_{2}(\mu)\right|_{\xi=\bar{b}}=\left.\varepsilon_{1} \bar{T}^{4}\right|_{\xi=\bar{b}}+\left.\rho_{1} \int_{0}^{1} J_{1}\left(\mu^{\prime}\right)\right|_{\xi=\bar{b}} d \mu^{\prime},
$$

We expect to come to the solution of the nonlinear problem (26), (27), (30)-(33), considering sequences of functions $\bar{T}^{(m)}(\xi), J_{1}^{(m)}(\xi, \mu), J_{2}^{(m)}(\xi, \mu), m=0,1,2, \ldots$, which satisfy the next iterative sequence of linear problems:

$$
\begin{aligned}
& \frac{d^{2} \bar{T}^{(m+1)}}{d \xi^{2}}=\Theta^{(m)}(\xi), \\
& \mu \frac{\partial J_{1}^{(m+1)}}{\partial \xi}+J_{1}^{(m+1)}= \Phi^{(m)}(\xi, \mu), \\
& \mu \frac{\partial J_{2}^{(m+1)}}{\partial \xi}+J_{2}^{(m+1)}= \Phi^{(m)}(\xi, \mu) \\
&\left.\frac{d \bar{T}^{(m+1)}}{d \xi}\right|_{\xi=\bar{b}}=\vartheta^{(m)}, \\
&\left.\bar{T}^{(m+1)}\right|_{\xi=-\bar{b}}=1, \\
&\left.J_{1}^{(m+1)}\right|_{\xi=-\bar{b}}=1, \\
&\left.J_{2}^{(m+1)}\right|_{\xi=\bar{b}}=\left.\varepsilon_{1}\left(\bar{T}^{(k)}\right)^{4}\right|_{\xi=\bar{b}} \\
& \quad+\left.2 \rho_{1} \int_{0}^{1} J_{1}^{(k)}\right|_{\xi=\bar{b}} \mu d \mu .
\end{aligned}
$$

Here we used the denotations:

$$
\begin{aligned}
& \Theta^{(m)}(\xi)=2 \frac{\bar{\kappa}}{\bar{\lambda}}\left(2\left(\bar{T}^{(m)}(\xi)\right)^{4}\right. \\
& \left.-\left(\int_{0}^{1} J_{1}^{(m)}(\xi, \mu) d \mu+\int_{-1}^{0} J_{2}^{(m)}(\xi, \mu) d \mu\right)\right), \\
& \Phi^{(m)}(\xi)=\bar{\kappa}\left(\bar{T}^{(m)}(\xi)\right)^{4}+\frac{1}{2} \bar{\sigma}\left(\int_{0}^{1} J_{1}^{(m)}\left(\xi, \mu^{\prime}\right) d \mu^{\prime}\right. \\
& \left.+\int_{-1}^{0} J_{2}^{(m)}\left(\xi, \mu^{\prime}\right) d \mu^{\prime}\right) . \\
& 9^{(m)}=\bar{\gamma}_{1}\left(\left.\bar{T}^{(m)}\right|_{\xi=\bar{b}}-\left.\bar{T}^{\mathrm{ext}}\right|_{\xi=\bar{b}}\right)+\left(1+n^{-2}\right) \\
& \cdot \bar{\lambda}^{-1} \varepsilon_{1}\left(\left.\bar{T}^{(m)}\right|_{\xi=\bar{b}}\right)^{4}-2 \varepsilon_{1}\left(1-\rho_{1}\right) \\
& \left.\cdot \bar{\lambda}^{-1} \int_{0}^{1} J_{1}^{(m)}(\mu)\right|_{\xi=\bar{b}} \mu d \mu
\end{aligned}
$$


Integrating ordinary differential equations (34)-(36) and subordinated their solutions to boundary conditions (37) and (38), we obtain

$$
\begin{aligned}
& \bar{T}^{(m+1)}(\xi)=\int_{-\bar{b}}^{\xi} \int_{-\bar{b}}^{z} \Theta^{(m)}(\zeta) d \zeta d z+\left(\vartheta^{(m)}\right. \\
& \left.\quad-\int_{-\bar{b}}^{b} \Theta^{(m)}(\xi) d \xi\right)(\xi+\bar{b})+1 \\
& J_{1}^{(m+1)}(\xi, \mu)=\int_{-\bar{b}}^{\xi} \frac{\Phi^{(m)}(z) e^{-(\xi-z) / \mu}}{\mu} d z+e^{-(\xi+\bar{b}) / \mu} \\
& J_{2}^{(m+1)}(\xi, \mu)=\int_{\bar{b}}^{\xi} \frac{\Phi^{(m)}(z) e^{-(\xi-z) / \mu}}{\mu} d z \\
& \quad+e^{-(\xi-\bar{b}) / \mu}\left(\varepsilon_{1}\left(\left.\bar{T}^{(m)}\right|_{\bar{y}=\bar{b}}\right)^{4}\right. \\
& \left.\quad+\left.2 \rho_{1} \int_{0}^{1} J_{1}^{(k)} \mu^{\prime} d \mu^{\prime}\right|_{\xi=\bar{b}}\right) .
\end{aligned}
$$

To avoid iterative integration, we approximate the solutions by linear finite elements. To do this we chose on the segment $[-\bar{b}, \bar{b}] N+1$ regular node points $\xi_{i}=$ $-\bar{b}+i \cdot 2 \bar{b} / N, i=\overline{0, N}$, and represent the functions $\bar{T}^{(m)}(\xi), J_{1}^{(m)}(\xi, \mu)$ and $J_{2}^{(m)}(\xi, \mu)$ by their node values: $\bar{T}_{i}^{(m)} \equiv$ $T^{(m)}\left(\xi_{i}\right), J_{1 i}^{(m)}(\mu) \equiv J_{1}^{(m)}\left(\xi_{i}, \mu\right), J_{2 i}^{(m)}(\mu) \equiv J_{2}^{(m)}\left(\xi_{i}, \mu\right)$. Approximating $\bar{T}^{(m)}(\xi), J_{1}^{(m)}(\xi, \mu)$ and $J_{2}^{(m)}(\xi, \mu)$ on the elements $\left[\xi_{i}, \xi_{i+1}\right], \quad i=\overline{0, N-1}$, by linear functions,

$$
\begin{aligned}
\bar{T}^{(m)}(\eta) & =\bar{T}_{i}^{(m)} \varphi_{1}(\eta)+\bar{T}_{i+1}^{(m)} \varphi_{2}(\eta), \\
J_{1}^{(m)}(\eta, \mu) & =J_{1 i}^{(m)}(\mu) \varphi_{1}(\eta)+J_{1 i+1}^{(m)}(\mu) \varphi_{2}(\eta), \\
J_{2}^{(m)}(\eta, \mu) & =J_{2 i}^{(m)}(\mu) \varphi_{1}(\eta)+J_{2 i+1}^{(m)}(\mu) \varphi_{2}(\eta),
\end{aligned}
$$

where

$$
\begin{aligned}
& \varphi_{1}(\eta)=\frac{1}{2}(1+\eta), \\
& \varphi_{2}(\eta)=\frac{1}{2}(1-\eta) .
\end{aligned}
$$

We replace the integrals in the right-hand sides of relations (42)-(44) with corresponding sums and obtain in the issue

$$
\begin{aligned}
& \bar{T}_{i}^{(m+1)}=\frac{\Delta^{2}}{4} \sum_{j=0}^{i-1}\left(2 \sum_{p=0}^{j}\left(\Theta_{p}^{(m)}+\Theta_{p+1}^{(m)}\right)-\Theta_{j}^{(m)}-\Theta_{j+1}^{(m)}\right) \\
& +\left(\vartheta_{i}^{(m)}-\frac{\Delta}{2} \sum_{j=0}^{i-1}\left(\Theta_{j}^{(m)}+\Theta_{j+1}^{(m)}\right)\right)\left(\xi_{i}+\bar{b}\right)+1 \\
& J_{1 i}^{(m+1)}(\mu)=\frac{e^{-\xi_{i} / \mu}}{\mu}\left[\sum_{j=1}^{i-1} \Phi_{j}^{(m)}\left(v_{j}(\mu)+w_{j-1}(\mu)\right)\right. \\
& \left.+\Phi_{0}^{(m)} v_{0}(\mu)+\Phi_{i}^{(m)} w_{i-1}(\mu)+\mu e^{-\bar{b} / \mu}\right] \\
& \quad+\Phi_{i}^{(m)} v_{i}(\mu)+\Phi_{N}^{(m)} w_{N-1}(\mu) \\
& \left.+\mu e^{\bar{b} / \mu}\left(\varepsilon_{1}\left(\bar{T}_{N}^{(m)}\right)^{4}+2 \rho_{1} \int_{0}^{1} J_{1 N}^{(m)}\left(\mu^{\prime}\right) \mu^{\prime} d \mu^{\prime}\right)\right\}
\end{aligned}
$$

Here,

$$
\begin{aligned}
v_{j}(\mu) & =-\frac{\mu}{\Delta}\left[(\Delta+\mu) e^{\xi_{j} / \mu}-\mu e^{\xi_{j} / \mu}\right], \\
w_{j}(\mu) & =\frac{\mu}{\Delta}\left[(\Delta-\mu) e^{\xi_{j+1} / \mu}+\mu e^{\xi_{j} / \mu}\right], \\
\Delta & \equiv \frac{2 b}{N} \\
\Theta_{j}^{(m)} & \equiv \Theta^{(m)}\left(\xi_{i}\right), \\
\Phi_{j}^{(m)} & \equiv \Phi^{(m)}\left(\xi_{i}\right) .
\end{aligned}
$$

\section{Numerical Study}

We tested a convergence of the iterative process (42)-(44) numerically, depending on chosen values of dimensionless parameters $\bar{b}, \bar{\lambda}, \bar{\kappa}, \bar{\sigma}, \varepsilon_{1}, \rho_{1}, \gamma_{1}$. The calculations were stopping when the criterion $\max \left\{\varepsilon_{T}, \varepsilon_{J}\right\} \leq \varepsilon$ was meted, where $\varepsilon$ is a given positive constant and

$$
\begin{aligned}
& \mathcal{E}_{T} \equiv \sqrt{\sum_{i=0}^{N}\left(\bar{T}_{i}^{(m+1)}-\bar{T}_{i}^{(m)}\right)^{2}}, \\
& \varepsilon_{J} \equiv \sqrt{\sum_{i=0}^{N}\left(\int_{0}^{1}\left(J_{1 i}^{(m+1)}(\mu)-J_{1 i}^{(m)}(\mu)\right) d \mu+\int_{-1}^{0}\left(J_{2 i}^{(m+1)}(\mu)-J_{2 i}^{(m)}(\mu)\right) d \mu\right)} .
\end{aligned}
$$


In Figure 1, we can see how the dimensionless intensity $J$ of radiation changes with coordinate $\xi$, depending on the direction of its incidence. The data for two different values of hemispherical emissivity $\varepsilon_{1}$ of the boundary $\mathbf{S}_{1}$ are presented in the figure.

In Figure 2 the distributions of dimensionless temperature $\bar{T}(\xi)$ and dimensionless radiation temperature $\bar{T}_{r}(\xi)$ in the layer are shown at the same two values of hemispherical emissivity $\varepsilon_{1}$. Radiation temperature is defined as $\bar{T}_{r}=\left(\bar{G}_{r} / 2\right)^{1 / 4}$, where $\bar{G}_{r}$ is dimensionless spatial density of radiation $\bar{G}_{r}(\xi)=\int_{0}^{1} J_{1}(\xi, \mu) d \mu+\int_{-1}^{0} J_{2}(\xi, \mu) d \mu$.

In Figure 3 the coordinate dependencies of dimensionless conduction heat flux $\bar{q}_{c}(\xi)=-\bar{\lambda} \partial \bar{T}(\xi) / \partial \xi$, radiation energy flux $\bar{q}_{r}(\xi)=\int_{0}^{1} J_{1}(\xi, \mu) \mu d \mu+\int_{-1}^{0} J_{2}(\xi, \mu) \mu d \mu$, and total flux $\bar{q}(\xi)=\bar{q}_{c}(\xi)+\bar{q}_{r}(\xi)$ are presented.

Figure 4 illustrates how the dimensionless energy flux $\bar{q}^{\text {out }}$, inflowing into external medium from the layer through boundary $S_{1}$, depends on boundary's emissivity $\varepsilon_{1}$. In considering the case the flux $\bar{q}^{\text {out }}$ contains three constituents: convective heat flux $\bar{q}_{\text {conv }}^{\text {out }}=\gamma_{1}\left(\left.\bar{T}\right|_{\xi=\bar{b}}-\left.\bar{T}^{\text {ext }}\right|_{\xi=\bar{b}}\right)$, energy flux $\bar{q}_{\mathbf{S}_{1}}^{\text {out }}=\varepsilon_{1} \bar{T}_{\xi=\bar{b}}^{4}$ emitted by the boundary $\mathbf{S}_{1}$, and energy flux $\bar{q}_{\mathbf{V}}^{\text {out }}=\left.\left(1-\varepsilon_{2}\right)\left(1-\rho_{2}\right) \int_{0}^{1} J_{1}(\mu)\right|_{\xi=\bar{b}} d \mu$ coming out of the volume V. The fluxes $\bar{q}_{\mathbf{S}_{1}}^{\text {out }}$ and $\bar{q}_{\mathrm{V}}^{\text {out }}$ are also shown in Figure 4 .

Figure 5 illustrates the influence of boundary's $\boldsymbol{S}_{1}$ emissivity $\varepsilon_{1}$ on directional dependence of dimensionless intensity $J_{\mathrm{V}}^{\text {out }}(\mu)=\left.\left(1-\varepsilon_{2}\right)\left(1-\rho_{2}\right) J_{1}(\mu)\right|_{\xi=\bar{b}}$ of radiation going out of the volume $\mathbf{V}$ through the boundary $\mathbf{S}_{1}$, where $\theta=\arccos (\mu)$ is a zenith angle of radiation propagation.

The data presented on the figures were obtained for the next values of dimensionless parameters: $\bar{b}=1.2, \bar{\lambda}=7, \bar{\kappa}=$ $0.1, \bar{\sigma}=0.9, \rho_{1}=0.2, n=1.65, \gamma_{1}=0.01, \bar{T}^{\text {ext }}=0.3$, $T_{0}=1000 \mathrm{~K}$. Conducted numerical studies show that, for the chosen values of the parameters, the iterative process is convergent up to optical thickness of the layer that equals 3 $(\bar{b}=1.5)$.

Analyzing Figures 1 and 2, we can conclude that there are substantial differences between values of conductive $\bar{T}$ and radiative $\bar{T}_{r}$ temperatures in the layer and between values of intensity of radiation incident in directions of positive $\mu \epsilon$ $(0,1)$ and negative $\mu \in(-1,0)$ hemispheres. It means that, at chosen values of the dimensionless parameters, the thermal state of the layer is far from a radiative equilibrium.

The data presented in Figures 1-3 show the substantial effect of surface radiative properties, in particular the boundary's hemispherical emissivity, on temperature field and radiation distribution in the layer. Figures 4 and 5 exhibit that these properties considerably affect the radiation emitted by the layer into external medium. It means that angular distribution of the radiation intensity emitting by the layer can be used as informative parameters for nondestructive determination of the volumetric and surface radiative properties of the medium.

\section{Conclusion}

The mathematical model for describing of combined conductive-radiative heat transfer in a dielectric layer which emits, absorbs, and scatters IR radiation both in the layer's volume and on its boundary has been considered. The layer is considered as thermodynamic system consisting of two interacting subsystems, the layer's medium and the layer's radiation, in correspondence to the fact that the heat transfer in the layer's volume is described by two interdependent processes, the heat conduction in the layer's medium and radiation transfer. Following this conception the heat conduction equation has been obtained proceeding from the equation of energy balance for layer's medium. In such form, HCE does not involve the radiation heat flux $\mathbf{q}_{r}$ but it contains the source-type terms taking into account the interaction of the layer's medium with the radiation subsystem. The boundary conditions for HCE also do not contain the radiation heat flux $\mathbf{q}_{r}$, but they contain the terms describing the interaction of the layer's medium and the radiation on the layer boundary. This interaction is caused by the surface radiation properties of layer's medium, being hemispherical, emissivity/absorptivity, and reflectivity.

Such formulation of the model enables determining the spatial-and-angular distributions of parameters of IR radiation, emitting by semitransparent mediums. This radiation usually contains the energy fluxes emitting both by the boundary and by interior of the layer. This feature of the model makes it perspective for utilization in inverse problems intended on noncontact determination of the temperature fields and/or volumetric and surface radiation properties of such mediums proceeding from the data, obtained by remote measuring of parameters of IR radiation, emitted by the object. Such data can be gathered with the use of modern high-precision measurement techniques, such as IR thermography.

A nonlinear stationary boundary-value problem for coupled integrodifferential heat conduction equation and radiation transfer equation for the layer, which exchanges by energy with external medium by convection and radiation, has been formulated. Features of the problem, depending on the layer's thickness, have been studied with the use of dimensional analysis. It was established that, in the case of optically thick layer, when the layer's thickness is much more of photon-free path, the problem becomes singularly perturbed one. In the inverse case of optically thin layer, the problem is regularly perturbed, and it becomes a regular (unperturbed) one, when the layer's thickness is of order of several photon's free paths.

An iterative method for solving of the unperturbed problem has been developed. The method reduces the initial nonlinear problem to the sequence of linear problems. Due to this the solutions for iterations were obtained in closed form. The conducted numerical studies show that the iterative processes is convergent up to value of optical thickness that equals 3 .

The method, in aggregate with the known diffusion approximation, can be used to implement an approach for solving of singularly perturbed problems and for quantitative analysis of the problem for conductive-radiative heat transfer in optically thick layer. Such approaches, consisting in considering of the singularly perturbed problem in the interior of 


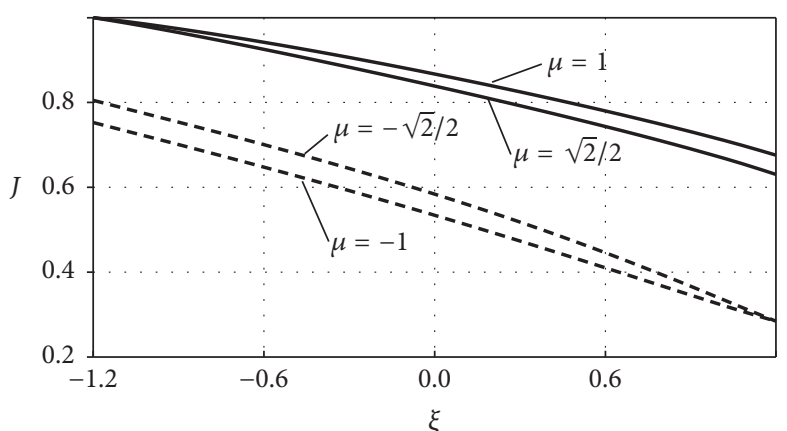

(a)

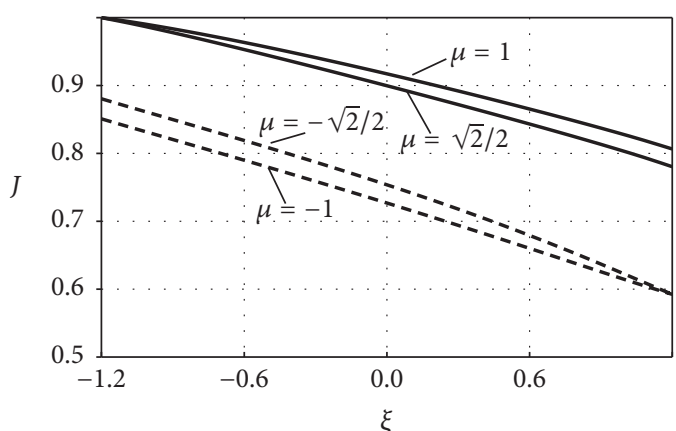

(b)

FIGURE 1: Dependencies of dimensionless intensity of radiation on spatial coordinate $\xi$ for different direction of its incidence at two values of hemispherical emissivity $\varepsilon_{1}$ : (a) $-\varepsilon=0.2$; (b) $\varepsilon=0.5$.

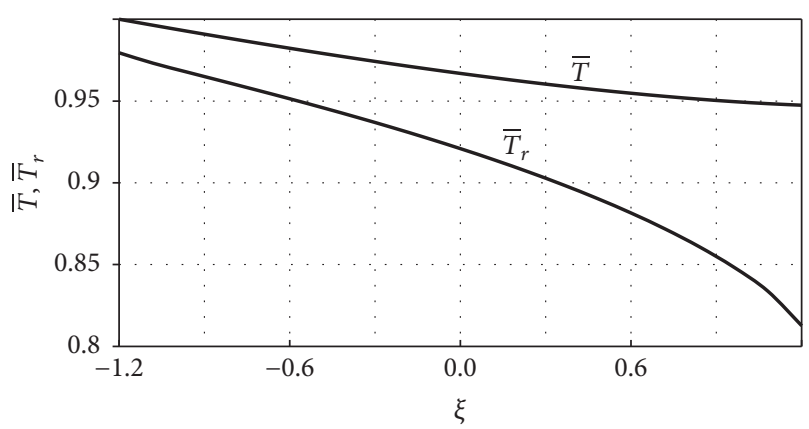

(a)

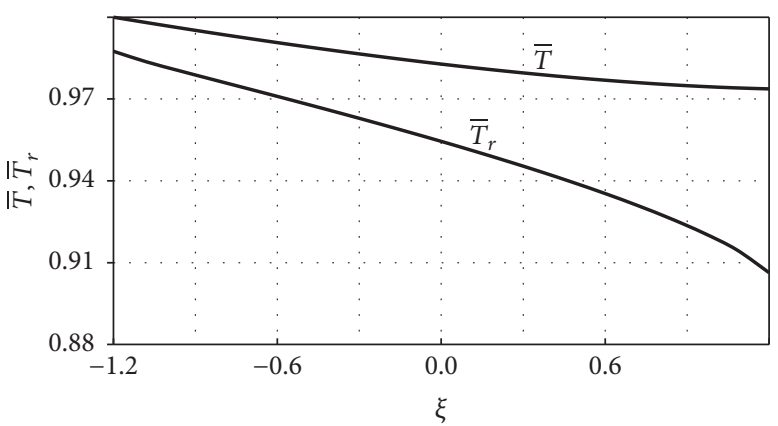

(b)

FIGURE 2: Dependencies of dimensionless temperature $\bar{T}$ and radiation temperature $\bar{T}_{r}$ on spatial coordinate for two different values of hemispherical emissivity $\varepsilon_{1}$ : (a) $-\varepsilon=0.2$; (b) $\varepsilon=0.5$.

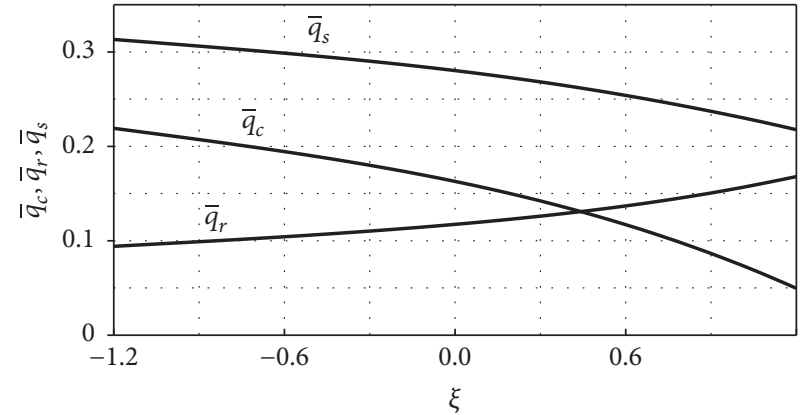

(a)

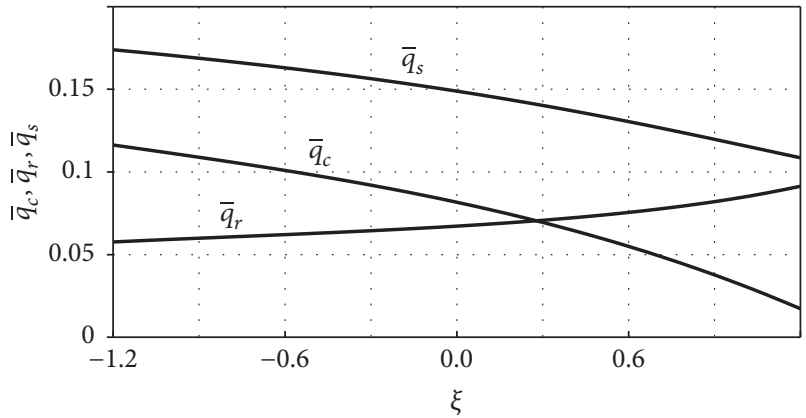

(b)

Figure 3: Dependencies of dimensionless conductive $\bar{q}_{r}$, radiative $\bar{q}_{r}$, and total $\bar{q}$ energy fluxes on coordinate $\xi$ for two different values of hemispherical emissivity $\varepsilon_{1}$ : (a) $-\varepsilon_{1}=0.2$; (b) $-\varepsilon_{1}=0.5$.

domain and in the boundary layer separately with consequent joining of the two obtained solutions, are known [10].

With the use of the developed method the temperature field and distribution of radiation intensity in the layer have been studied. The obtained numerical results exhibit substantial dependence of the IR radiation, emitted by the layer into the external medium, on the temperature field in the layer and on its radiative properties. This makes possible to expect that developed model and method can be used to formulate and solve inverse problems for noncontact temperature testing in dielectrics and for nondestructive determination of its radiation properties (volumetric and surface) on the base of the data obtained by measuring of IR radiation emitted by the layer. 


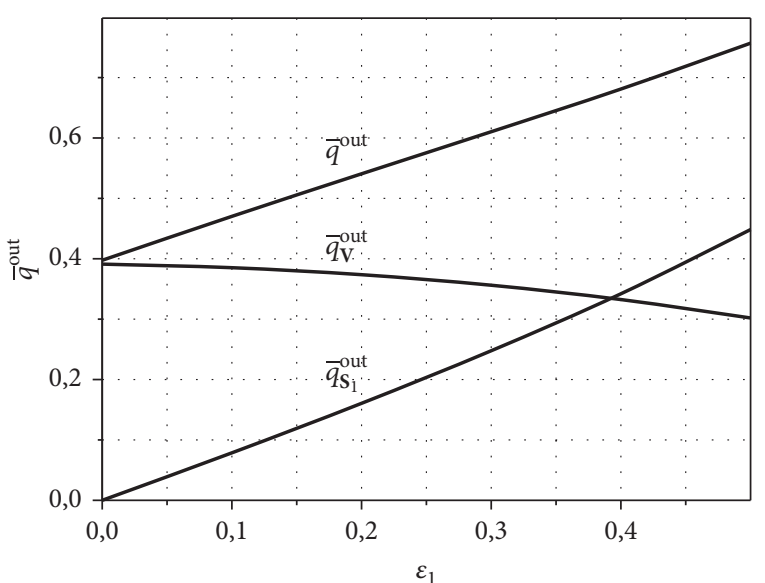

Figure 4: Dependencies of the dimensionless energy flux $\bar{q}^{\text {out }}$ outgoing from the layer through boundary $\mathbf{S}_{1}$ and its constituents $\bar{q}_{\mathrm{s}_{1}}^{\text {out }}, \bar{q}_{\mathrm{v}}^{\text {out }}$ on emissivity $\varepsilon_{1}$.

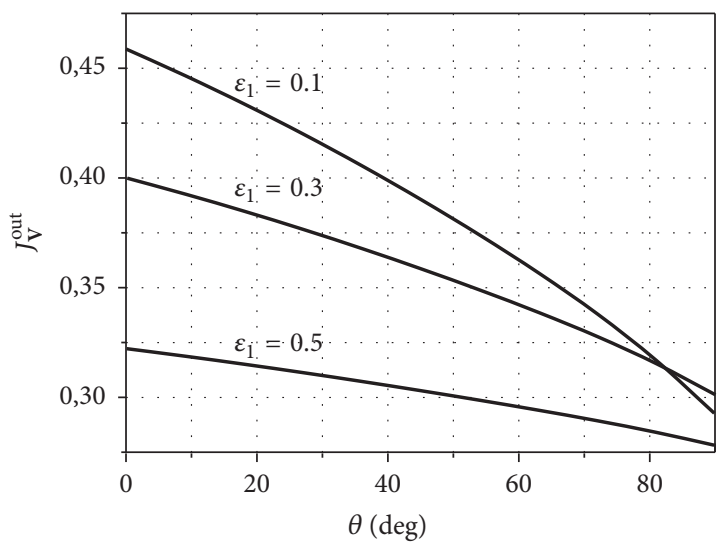

FIGURE 5: Directional dependence of $J_{\mathrm{V}}^{\text {out }}$ for different value of boundary's $\boldsymbol{S}_{1}$ emissivity $\varepsilon_{1}$.

\section{Conflicts of Interest}

The authors declare that there are no conflicts of interest regarding the publication of this paper.

\section{References}

[1] M. N. Ozisik, Radiative Transfer and Interactions with Conduction And Convection, Wiley, New York, NY, USA, 1973.

[2] A. Bejan and A. D. Kraus, Heat Transfer Handbook, John Wiley \& Sons, New York, NY, USA, 2003.

[3] W. M. Rohsenow, Handbook of Heat Transfer, McGraw-Hill, New York, NY, USA, 3rd edition, 1998.

[4] C. E. Siewert, J. R. Maiorino, and M. N. Ozisik, "The use of the $\mathrm{F}_{N}$ method for radiative transfer problems with reflective boundary conditions," Journal of Quantitative Spectroscopy \& Radiative Transfer, vol. 23, pp. 545-573, 1980.

[5] M. P. Mengüç and R. K. Iyer, "Modeling of radiative transfer using multiple spherical harmonics approximations," Journal of Quantitative Spectroscopy and Radiative Transfer, vol. 39, no. 6, pp. 445-461, 1988.
[6] T. Heping, B. Maestre, and M. Lallemand, "Transient and steady-state combined heat transfer in semi-transparent materials subjected to a pulse or a step irradiation," Journal of Heat Transfer, vol. 113, no. 1, pp. 166-173, 1991.

[7] L. H. Liu and H. P. Tan, "Non-fourier effects on transient coupled radiative-conductive heat transfer in one-dimensional semitransparent medium subjected to a periodic irradiation," Journal of Quantitative Spectroscopy and Radiative Transfer, vol. 71, no. 1, pp. 11-24, 2001.

[8] L. H. Liu, H. P. Tan, and T. W. Tong, "Non-Fourier effects on transient temperature response in semitransparent medium caused by laser pulse," International Journal of Heat and Mass Transfer, vol. 44, no. 17, pp. 3335-3344, 2001.

[9] L. H. Liu and H. P. Tan, "Transient temperature response in semitransparent variable refractive index medium subjected to a pulse irradiation," Journal of Quantitative Spectroscopy and Radiative Transfer, vol. 83, no. 3-4, pp. 333-344, 2004.

[10] A. H. Nayfeh, Problems in Perturbation, John Wiley \& Sons, New York, NY, USA, 1985.

[11] C. Huang and Y. Zhang, "Calculation of high-temperature insulation parameters and heat transfer behaviors of multilayer insulation by inverse problems method," Chinese Journal of Aeronautics, vol. 27, no. 4, pp. 791-796, 2014.

[12] K. Daryabeigi, G. R. Cunnington, and J. R. Knutson, “Combined heat transfer in high-porosity high-temperature fibrous insulation: theory and experimental validation," Journal of Thermophysics and Heat Transfer, vol. 25, no. 4, pp. 536-546, 2011.

[13] M. Maerefat and A. Esmaili, "Analysis of combined conductionradiation heat transfer in multilayer insulations," Amirkabir University of Technology, vol. 46, no. 1, pp. 17-19, 2014.

[14] R. Usamentiaga, P. Venegas, J. Guerediaga, L. Vega, J. Molleda, and F. G. Bulnes, "Infrared thermography for temperature measurement and non-destructive testing," Sensors, vol. 14, no. 7, pp. 12305-12348, 2014.

[15] A. C. Murariu, A. V. Bîrdeanu, R. Cojocaru et al., "Application of Thermography in Materials Science and Engineering," in Infrared Thermography, pp. 27-52, InTech, 2012. 


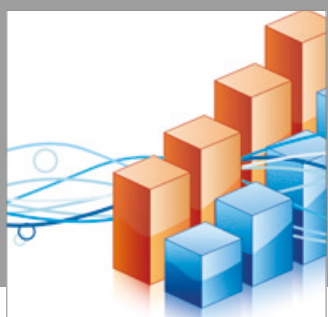

Advances in

Operations Research

vatersals

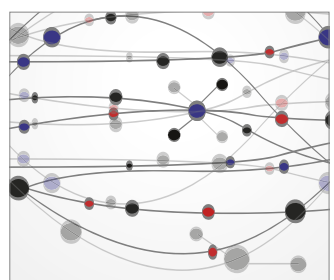

\section{The Scientific} World Journal
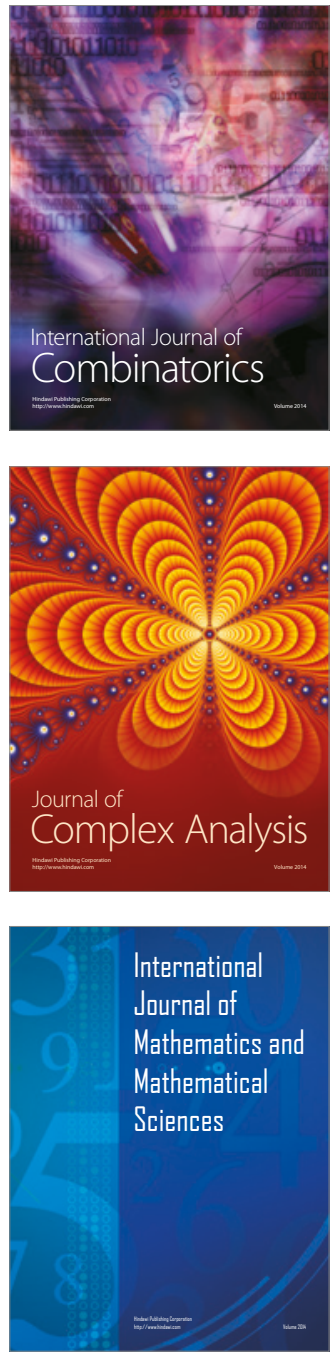
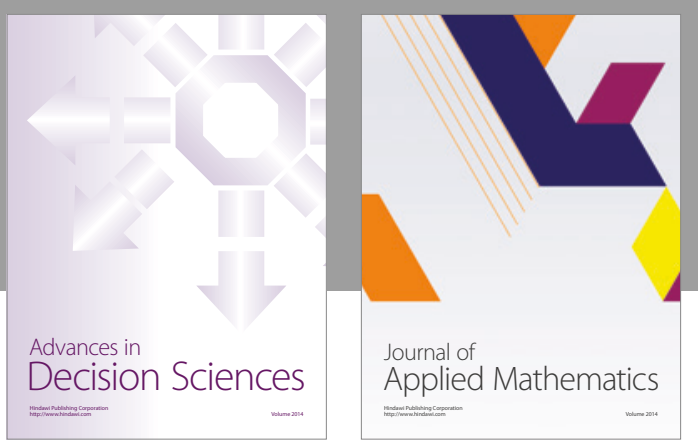

Algebra

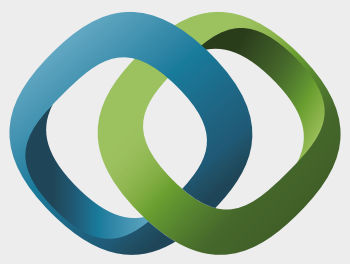

\section{Hindawi}

Submit your manuscripts at

https://www.hindawi.com
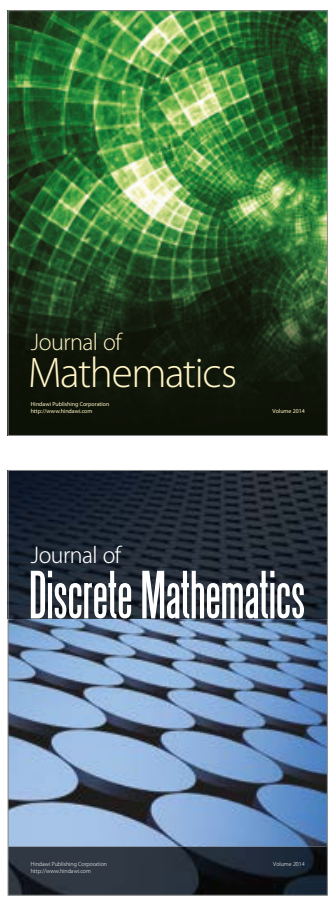

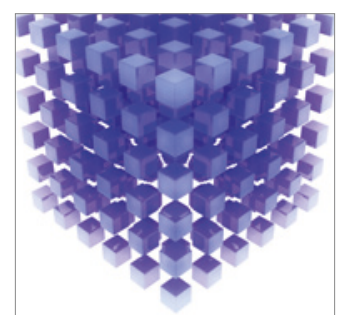

Mathematical Problems in Engineering
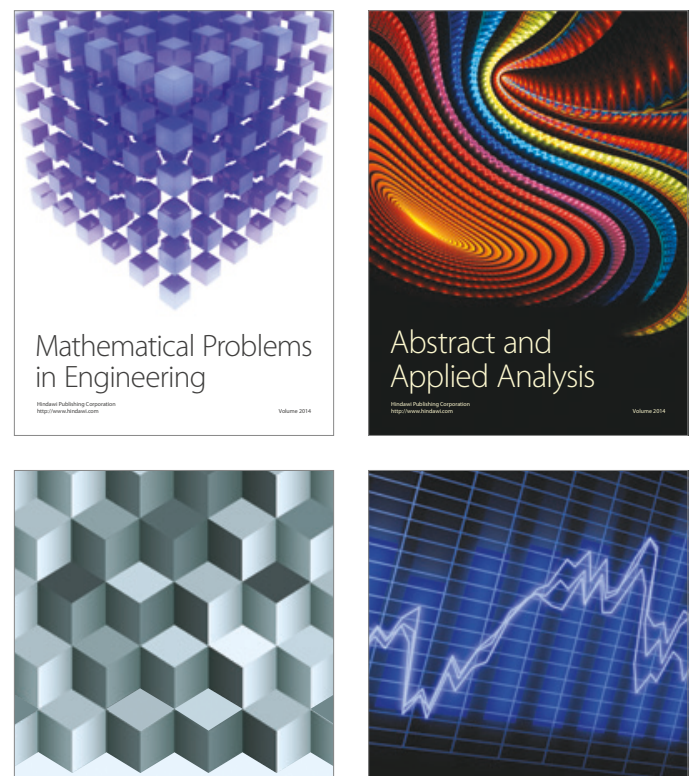

Journal of

Function Spaces

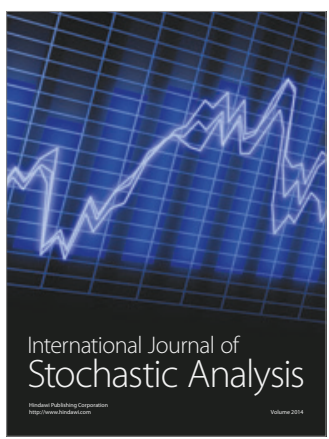

Probability and Statistics
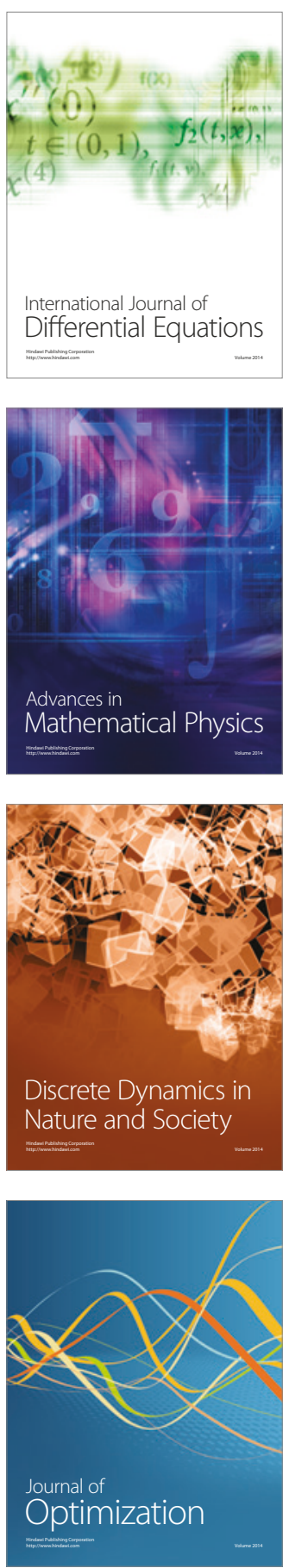